\title{
Refinando Dados Espaciais para a Conservação da Biodiversidade
}

\author{
Refining Spatial Data for Biodiversity Conservation
}

\author{
Priscila Lemes ${ }^{1 *}$, Frederico Augusto Martins Valtuille Faleiro1, Geiziane Tessarolo² \& \\ Rafael Dias Loyola ${ }^{1}$
}

\begin{abstract}
${ }^{1}$ Laboratório de Biogeografia da Conservação, Departamento de Ecologia, Instituto de Ciências Biológicas - ICB Universidade Federal de Goiás - UFG, Goiânia, GO, Brazil

${ }^{2}$ Laboratório de Ecologia Teórica e Síntese, Departamento de Ecologia, Instituto de Ciências Biológicas - ICB, Universidade Federal de Goiás - UFG, Goiânia, GO, Brazil
\end{abstract}

A conservação in situ é a pedra angular da biologia da conservação, i.e., proteger populações em locais onde estas ocorrem naturalmente, ainda hoje, se apresenta globalmente como a estratégia de conservação mais viável e econômica (Loucks et al. 2008). Entretanto, desde a escala regional até a global, a eficiência das áreas protegidas em representar os diversos atributos da biodiversidade ainda é baixa (Rodrigues et al. 2004). Fundamentalmente, essa ineficiência advém do fato de que o estabelecimento de novas reservas não tem base científica, importando mais, na prática, a vontade política e a oportunidade imediata. Como alternativa a esse tipo de priorização surgiu a ciência do planejamento sistemático para a conservação (Margules \& Pressey 2000), que visa propor o melhor conjunto de locais para a conservação e manejo da biodiversidade dentre aqueles disponíveis, satisfazendo princípios-chave como abrangência, adequação, representatividade e eficiência (Wilson et al. 2009).

O desenvolvimento e a implementação política de planos de conservação que atendam a esses princípios representa um ganho de qualidade e eficiência na criação e manejo de reservas. Contudo, o planejamento sistemático necessita de uma série de dados para seu desenvolvimento, sendo os dados espaciais extremamente importantes e necessários. Particularmente em países megadiversos, dados acurados sobre a distribuição geográfica das espécies são escassos e difíceis de serem obtidos. Tradicionalmente, o planejamento para a conservação nesses países é feito com base em dados de baixa qualidade, normalmente disponíveis com mapas de extensão de ocorrência de espécies.

*Send correspondence to: Priscila Lemes

Departamento de Ecologia, Instituto de Ciências Biológicas,

Universidade Federal de Goiás - UFG,

CP 131, CEP 74001-970, Goiânia, GO, Brasil

e-mail: priscila.lemes.azevedo@gmail.com

\section{O Demônio em Forma de Incerteza: o Déficit Wallaceano}

Um dos gargalos mais apertados no momento do planejamento para a conservação é o fato de que, para uma infinidade de espécies, as distribuições geográficas são pouco conhecidas e possuem inúmeras lacunas - problema conhecido como déficit Wallaceano (Whittaker et al. 2005), em homenagem a Alfred R. Wallace, o pai da zoogeografia. Diante dessa incerteza sobre onde as espécies ocorrem, usa-se, normalmente, uma inferência sobre tal distribuição. Todavia, ao inferir a distribuição de uma espécie, dois erros fundamentais podem ser cometidos. O primeiro é o erro de omissão: omite-se a ocorrência da espécie em uma determinada área, quando ela ocorre ali de fato. O segundo é o erro de comissão (ou sobreprevisão): pressupõe-se a ocorrência da espécie em um local onde ela não ocorre verdadeiramente. Tais erros minam o planejamento para a conservação e geram cenários de incerteza que atormentam os tomadores de decisão. O pior deles, chamemo-lo de cenário da "extinção por exagero", é considerar (devido a um erro de comissão) que uma espécie será protegida em um local caso o mesmo seja demarcado como uma reserva, quando ela não ocorre ali. Esse erro de julgamento poderia levar à extinção de populações dessa espécie em regiões fora da reserva, onde a espécie de fato ocorre. O segundo pior cenário é o da "extinção por ignorância". Nesse caso, o número de locais disponíveis para a implementação da ação de conservação é substancialmente reduzido, pois a ocorrência da espécie nos mesmos é desconhecida (devido a um erro de omissão), levando a uma menor eficiência do planejamento e possível extinção das populações desconhecidas. Esses problemas crescem exponencialmente quando o planejamento é executado para inúmeras espécies - as vezes milhares - ao mesmo tempo (e.g. Loyola et al. 2009). 


\section{Como Estimar a Amplitude de Distribuição Geográfica de uma Espécie?}

Atualmente, existem três alternativas básicas para estimar a distribuição geográfica de uma espécie a partir de dados espaciais pré-existentes (ver Boitani et al. 2011 para uma discussão recente): 1) o uso de registros de ocorrência de indivíduos de uma espécie, obtidos em campo ou a partir de coleções abrigadas em museus; 2 ) o uso de mapas de extensão de ocorrência, disponíveis em bases de dados acessíveis na internet; e 3) o uso de mapas preditivos de distribuição, gerados a partir de modelos de distribuição de espécies. Todos esses dados possuem vantagens e desvantagens se considerarmos sua aplicação para a conservação.

De maneira geral, registros de ocorrência são bastante enviesados. Há inúmeros problemas associados à amostragem em amplas escalas geográficas (e.g. continental), há problemas relacionados à coleta per se e relatados amiúde pela literatura pertinente (e.g. coletas em locais de fácil acesso ao coletor e próximos a estradas abundam) e há pouca ou nenhuma informação sobre a ausência real de indivíduos e populações - não se faz um inventário para relacionar as espécies que não ocorrem em um local, senão aquelas que ocorrem. Devido a isso, esses dados possuem baixos erros de comissão e altos erros de omissão, embora tais problemas sejam dependentes do esforço amostral.

Mapas de extensão de ocorrência, em contrapartida, são polígonos espacializados, que tendem a incorrer em altos erros de comissão (falsas presenças) devido à interpolação geográfica de registros de ocorrência. $\mathrm{O}$ procedimento lógico de elaboração destes dados envolve mapear os registros de que presumidamente encontram-se na porção mais periférica da região de ocorrência de uma espécie e usar uma interpolação para delinear os limites da distribuição desta espécie. Existem, contudo, diferentes maneiras para a definição do contorno da distribuição. Assim, a interpolação que gera o polígono dentro do qual os registros se encontram pode ser estritamente baseada em dados de ocorrência (e.g. Polígono Convexo Mínimo) - ou seja, uma abordagem definida pelos próprios dados disponíveis (data-driven) - mas também mais informal e baseada em diferentes graus de conhecimento das condições ambientais (e.g. limites que seguem condições ambientais inóspitas para a espécie), ou ainda na opinião de especialistas (e.g. limites que excluem áreas onde a espécie não ocorre por razões históricas) - ou seja, uma abordagem definida pelo conhecimento prévio (knowledge-driven). É possível que tais mapas sejam ainda gerados por uma abordagem que reúne os dois métodos acima. A grande vantagem dos mapas de extensão de ocorrência reside no fato de que esses mapas, por serem generalizações espaciais da distribuição geográfica das espécies, estão disponíveis em escala global para uma série de grupos taxonômicos (especialmente para os vertebrados). Isso permite o delineamento de planos de conservação mesmo em regiões cuja disponibilidade de dados espaciais é parca. A desvantagem desses mapas, além dos altos erros de comissão já mencionados, reside na incerteza envolvida no uso da extensão de ocorrência. Tal incerteza é bastante difícil de ser avaliada, em função da subjetividade implícita ao desenho do polígono (e.g. escala original em que foi desenhado o mapa e os critérios adotados para sua delimitação). Na maioria das vezes tais subjetividades sequer são reportadas. Além disso, via de regra estes mapas não são validados.

Finalmente, mapas preditivos de distribuição, species distribution models - SDMs, (ver Franklin 2009 para uma revisão atual) vêm sendo usados como dados de entrada em análises de conservação, principalmente devido ao aumento crescente da disponibilidade de dados espaciais como os mencionados anteriormente, de Sistemas de Informação Geográfica (SIG), da capacidade computacional dos processadores domésticos e da existência de programas específicos para a modelagem. Apesar de sua suposta sofisticação, a aplicação desses modelos em conservação (assim como para outros usos) padece de problemas básicos como a ausência de consenso sobre a melhor maneira de validá-los (principalmente com uma validação biológica empírica e não apenas estatística) e a elevada incerteza associada aos mesmos (ver Diniz-Filho et al. 2009). Fontes de incerteza existem inúmeras (origem dos dados, algoritmo usado na modelagem, combinação de variáveis usadas para calibrar os modelos, métodos de validação, modelos climáticos etc.) e o busílis reside no fato de que, atualmente, não é possível comparar de maneira definitiva a qualidade de dois ou mais modelos (mas veja Araújo \& New 2007 para uma alternativa paliativa a esses problemas). Isso sem adicionar o fato de que os dados brutos para a modelagem vêm de registros de ocorrência ou mapas de extensão de ocorrência cujas limitações já foram aqui expostas. Portanto, sua qualidade permanece ainda dependente da qualidade e quantidade de registros de ocorrência - toda discussão em contrário é simplesmente debalde. Entretanto, essa constatação reforça a importância fundamental de investimentos em inventários com desenho amostral cuidadosamente planejado.

\section{A "Síndrome de Gabriela"}

Por que usamos dados como os mencionados acima em análises de conservação afinal? A resposta óbvia e ingênua para essa pergunta é embaraçosamente simples: porque, muitas vezes, essa é a única forma de fazer algum tipo de planejamento. Especialmente em regiões com alta biodiversidade, pobres em dados e com deficiências na proteção de espécies (países tropicais e emergentes, em sua maioria), registros de ocorrência são escassos e polígonos de distribuição constituem a única informação espacial sobre a amplitude de distribuição das espécies. Em escala global, por exemplo, análises com dados espaciais extremamente refinados tornam-se simplesmente impossíveis de serem conduzidas. A pergunta fundamental, portanto, e de resposta não trivial, é outra: devemos manter a popular e 
inercial "síndrome de Gabriela" (imortalizada pela modinha de Dorival Caymmi, cujo refrão diz: "Eu nasci assim, eu cresci assim, eu sou mesmo assim, vou ser sempre assim...") ou, diante dos desafios e da crise atual de biodiversidade, buscaremos soluções simples e/ou criativas para o problema?

O uso de modelos de distribuição de espécies é, portanto, extremamente promissor. Contudo, análises de conservação baseadas nos mesmos devem ser extremamente avaliadas sob o olhar crítico da falta de consenso sobre a precisão e acurácia desses modelos. Minimamente, as incertezas associadas a eles devem ser incluídas na análise como uma informação a mais para o tomador de decisão (ver Wilson 2010). Ainda assim, o uso de modelagem de distribuição é uma alternativa complexa, dependente de especialistas com amplo referencial acadêmico e requer extenso treinamento para sua aplicação de forma coerente e cuidadosa. Assim, para o especialista em áreas protegidas, o conservacionista de uma ONG ou um tomador de decisão que está diretamente engajado em um planejamento regional aplicável para a conservação, o uso de tais modelos é ainda proibitivo.

\section{Uma Alternativa Simples e Direta para Conservacionistas e Acadêmicos Atuantes}

Recentemente, tem-se sugerido um refinamento dos mapas de extensão de ocorrência por variáveis ambientais que determinam, grosso modo, a ocorrência das populações de uma espécie (ver Rondinini et al. 2011 para um exemplo global). Essas variáveis costumam ser o habitat preferencial da espécie, cobertura vegetal, variação altitudinal e a presença e extensão de corpos d'água. A idéia básica por trás desse estratagema é a "filtragem" de um dado espacial grosseiro (mapa de extensão de ocorrência) para a produção de um "modelo ou mapa de hábitat adequado", habitat suitability model - HSM, mais condizente com a realidade (Rondinini et al. 2011).

O uso desses mapas "filtrados” tem inúmeras vantagens: são relativamente simples de serem confeccionados, são baseados em SIG, os dados para sua criação encontram-se normalmente disponíveis (mapas, imagens de satélite, fotos aéreas) e os mapas finais podem ser refinados ainda mais, caso necessário. Em teoria, mapas de hábitat adequado reduzem os erros de comissão, pois retiram as porções dos polígonos que não contém áreas ambientalmente adequadas para a ocorrência e sobrevivência das populações de uma espécie. Além disso, essa "filtragem por habitat" gera "pseudoausências" mais reais, em relação àquelas geradas por algoritmos de inteligência artificial, empregados na modelagem de distribuição de espécies. Cabe apenas ressaltar que embora boa parte dos dados para a criação desses modelos encontre-se disponível, a informação sobre o que deve ser excluído da distribuição da espécie ou sobre o que constitui o hábitat inadequado nem sempre é fácil de ser obtida e permanece a dependência de conhecimento prévio ou especializado para que a modelagem possa ser realizada a contento.

Recentemente, Rondinini et al. (2011) criaram mapas de hábitat adequado para mais de 5.000 espécies de mamíferos terrestres, filtrando mapas de extensão de ocorrência por variávies como cobertura vegetal, elevação e características hidrológicas do terreno. Os novos mapas foram produzidos a uma resolução espacial de $300 \mathrm{~m}$, algo extremamente fino para uma avaliação global. A construção desses mapas permitiu uma análise detalhada dos padrões atuais de riqueza de espécies e de quanto os mapas de extensão de ocorrência foram refinados por meio dessa abordagem. Em outro estudo prévio, tais mapas foram usados em um exercício de priorização de áreas para a conservação de vertebrados africanos (Rondinini et al. 2005) e poderiam ser igualmente utilizados para a avaliação de outros aspectos da biodiversidade, como os avaliados por Carvalho et al. (2010) e Safi et al. (2011).

\section{Problema Resolvido?}

Infelizmente, nem tudo são flores. Mapas de hábitat adequado também estão sujeitos a críticas e têm limitações que precisam ser consideradas antes de sua aplicação imediata em um planejamento sistemático para a conservação (Rodrigues 2011).

Em primeiro lugar, informações sobre a história natural das espécies, como por exemplo, uma descrição suficiente sobre seu hábitat preferencial não são exatamente fáceis de se obter, principalmente para todas as espécies incluídas no planejamento. Descrições muito gerais (e.g. áreas abertas) não são muito úteis, ao passo que descrições extremamente detalhadas (e.g. margens de riachos de cabeceira, com substrato de cascalho e inclinação de terreno próxima à de $\left.13,5^{\circ}\right)$ jamais serão capturadas por dados georreferenciados de cobertura vegetal, por exemplo.

Um segundo entrave reside na resposta espécie-específica das populações, que sé difícil de se prever. Até que ponto a especialização intraespecífica e intraindividual tornam a associação do hábitat a uma espécie um exercício de generalização distante da realidade ao longo de uma vasta extensão geográfica? Espécies podem se beneficiar com impactos antrópicos específicos? Como incluir esse tipo de resposta em modelos (mapas) corrigidos por extensão de hábitat adequado? Como lidar com a incerteza na própria criação do mapa de extensão de ocorrência (como mencionado anteriormente)? De fato, essas perguntas permanecem sem resposta, assim como aquelas sobre a verdadeira eficácia desse tipo de abordagem em relação aos modelos de distribuição de espécies. Provavelmente, a resposta para isso jaz em uma interação entre a escala do planejamento e o objetivo do mesmo. Em escalas de grande extensão geográfica, mapas de hábitat adequado podem ser particularmente úteis para um planejamento mais fidedigno (ver, contudo, Rodrigues 2011). Em escalas de menor extensão geográfica, modelos adequados de 
distribuição de espécies podem ser uma ferramenta poderosa para aplicação em conservação, sobretudo quando têm sua incerteza mensurada, espacialmente mapeada e ponderada pelo planejador (Diniz-Filho et al. 2009; Wilson 2010).

De qualquer maneira, independente da escala do planejamento, uma discussão ainda mais básica sobre a qualidade dos dados de entrada do modelo (e.g. dados de ocorrência bem representativos e menos enviesados, informação sobre habitat adequado/inadequado de alta qualidade) permanece como central para a resolução do problema aqui apresentado. Permanece em aberto também a questão sobre o que é mais viável hoje em dia: boas bases de dados com registros de ocorrência ou uma boa base de conhecimento espécie-específico sobre o que é e o que não é habitat. Como expusemos nesse artigo, infelizmente não existem atalhos mágicos para refinar dados espaciais sobre a distribuição de espécies, com vistas à sua aplicação no planejamento para a conservação. Estudos futuros dirão o quanto o uso de mapas de hábitat adequado faz diferença no planejamento, acelerando a tomada de decisão ou ajudando na manutenção das populações em ambiente natural.

\section{Agradecimentos}

PL e FAMVF são bolsistas de doutorado e de mestrado do $\mathrm{CNPq}$, respectivamente. GT é bolsista de doutorado da CAPES. A pesquisa de RDL é financiada pelo CNPq (processos 475886/2009-7 e 563621/2010-9), CAPES (processo 012/2009) e MCT/Rede CLIMA.

\section{Referências}

Araújo MB \& New M, 2007. Ensemble forecasting of species distributions. Trends in Ecology and Evolution, 22:42-47. http://dx.doi.org/10.1016/j.tree.2006.09.010

Boitani L et al., 2011. What spatial data do we need to develop global mammal conservation strategies? Philosophical Transactions of the Royal Society of London Series B, 366:2623-2632. PMid:21844041. http://dx.doi.org/10.1098/ rstb.2011.0117

Carvalho RA et al., 2011. Drafting a Blueprint for Functional and Phylogenetic Diversity Conservation in the Brazilian Cerrado. Natureza \& Conservação, 8:171-176. http://dx.doi. org/10.4322/natcon.00802012

Diniz-Filho JAF et al., 2009. Partitioning and mapping uncertainties in ensembles of forecasts of species turnover under climate change. Ecography, 32:897-906. http://dx.doi. org/10.1111/j.1600-0587.2009.06196.x
Franklin J, 2009. Mapping Species Distributions: Spatial Inference and Prediction. Cambridge: Cambridge University Press. 320 p.

Margules CR \& Pressey RL, 2000. Systematic conservation planning. Nature, 405:243-253. PMid:10821285. http:// dx.doi.org/10.1038/35012251

Loucks C et al., 2008. Explaining the global pattern of protected area coverage: relative importance of vertebrate biodiversity, human activities and agricultural suitability. Journal of Biogeography, 35:1337-1348. http://dx.doi. org/10.1111/j.1365-2699.2008.01899.x

Loyola RD et al., 2009. Key Neotropical ecorregions for conservation of terrestrial vertebrates. Biodiversity and Conservation, 18:2017-2031. http://dx.doi.org/10.1007/ s10531-008-9570-6

Rodrigues ASL et al., 2004. Effectiveness of the global protected area network in representing species diversity. Nature, 428:640-643. PMid:15071592. http://dx.doi. org/10.1038/nature02422

Rodrigues ASL, 2011. Improving coarse species distribution data for conservation planning in biodiversity-rich, data-poor, regions: no easy shortcuts. Animal Conservation, 14:108-110. http://dx.doi.org/10.1111/j.1469-1795.2011.00451.x

Rondinini C, Stuart S \& Boitani L, 2005. Habitat suitability models and the shortfall in Conservation Planning for African Vertebrates. Conservation Biology, 19:1488-1497. http://dx.doi.org/10.1111/j.1523-1739.2005.00204.x

Rondinini C et al., 2011. Global habitat suitability models of terrestrial mammals. Philosophical Transactions of the Royal Society of London Series B, 366:2633-2641. P Mid:21844042. http://dx.doi.org/10.1098/rstb.2011.0113

Safi K et al., 2011. Understanding global patterns of mammalian functional and phylogenetic diversity. Philosophical Transactions of the Royal Society of London Series B, 366:2536-2544. PMid:21807734. http://dx.doi. org/10.1098/rstb.2011.0024

Wilson KA, 2010. Dealing with data uncertainty in conservation planning. Natureza \& Conservação, 8:145-150. http://dx.doi. org/10.4322/natcon.00802007

Wilson KA, Cabeza M \& Klein CJ, 2009. Fundamental Concepts of Spatial Conservation Prioritization. In: Moilanen A, Wilson KA \& Possingham HP (eds.). Spatial Conservation Prioritization. New York: Oxford University Press. p. 16-27.

Whittaker RJ et al., 2005. Conservation Biogeography: assessment and prospect. Diversity and Distributions, 11:3-23. http:// dx.doi.org/10.1111/j.1366-9516.2005.00143.x

Received: September 2011 First Decision: October 2011 Accepted: October 2011 